\title{
Direct imaging of tunable crystal surface structures of MOF MIL-101 using high-resolution electron microscopy
}

Xinghua Li, ${ }^{1}$ Jianjian Wang, ${ }^{2}$ Xin Liu, ${ }^{3}$ Lingmei Liu, ${ }^{1}$ Dongkyu Cha, ${ }^{4}$ Xinliang Zheng, ${ }^{5}$ Ali A. Yousef, ${ }^{4}$ Kepeng Song, ${ }^{1}$ Yihan Zhu, ${ }^{6}$ Daliang Zhang, ${ }^{2, *}$ Yu Han ${ }^{1, *}$

${ }^{1}$ King Abdullah University of Science and Technology (KAUST), Physical Sciences and Engineering Division, Advanced Membranes and Porous Materials Center, Thuwal 23955-6900, Saudi Arabia

2 Multi-scale Porous Materials Center, Institute of Advanced Interdisciplinary Studies, Chongqing University, Chongqing 400044, P. R. China

3 School of Chemistry, State Key Laboratory of Fine Chemicals, Dalian University of Technology, Dalian, 116024, P. R. China.

${ }^{4}$ Saudi Aramco, EXPEC Advance Research Center, Dhahran 31311, Saudi Arabia

5 School of Physics, Northwest University, Xi'an, 710069, P. R. China

${ }^{6}$ Department of Chemical Engineering, Zhejiang University of Technology, Hangzhou 310014, P.R. China

Correspondence to: daliang.zhang@cqu.edu.cn; yu.han@kaust.edu.sa

This PDF includes 8 Supporting figures (Figures S1-S8), a computational section, and a list of supporting references. 

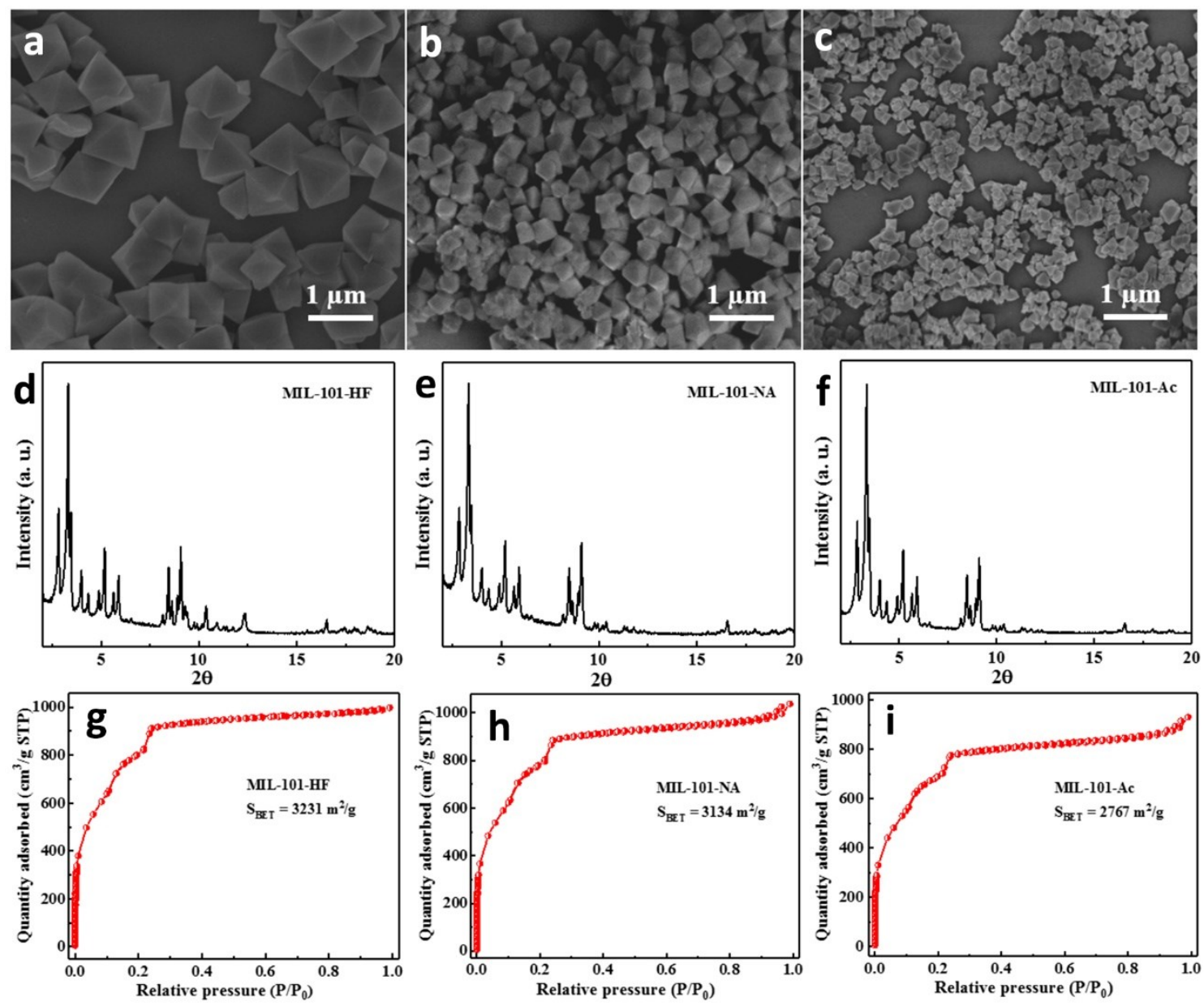

Figure S1. SEM images of (a) MIL-101-HF, (b) MIL-101-NA and (c) MIL-101-Ac. PXRD patterns of (d) MIL-101-HF, (e) MIL-101-NA and (f) MIL-101-Ac. $\mathrm{N}_{2}$ adsorption isotherms of (g) MIL-101-HF, (h) MIL-101-NA and (i) MIL-101-Ac. 


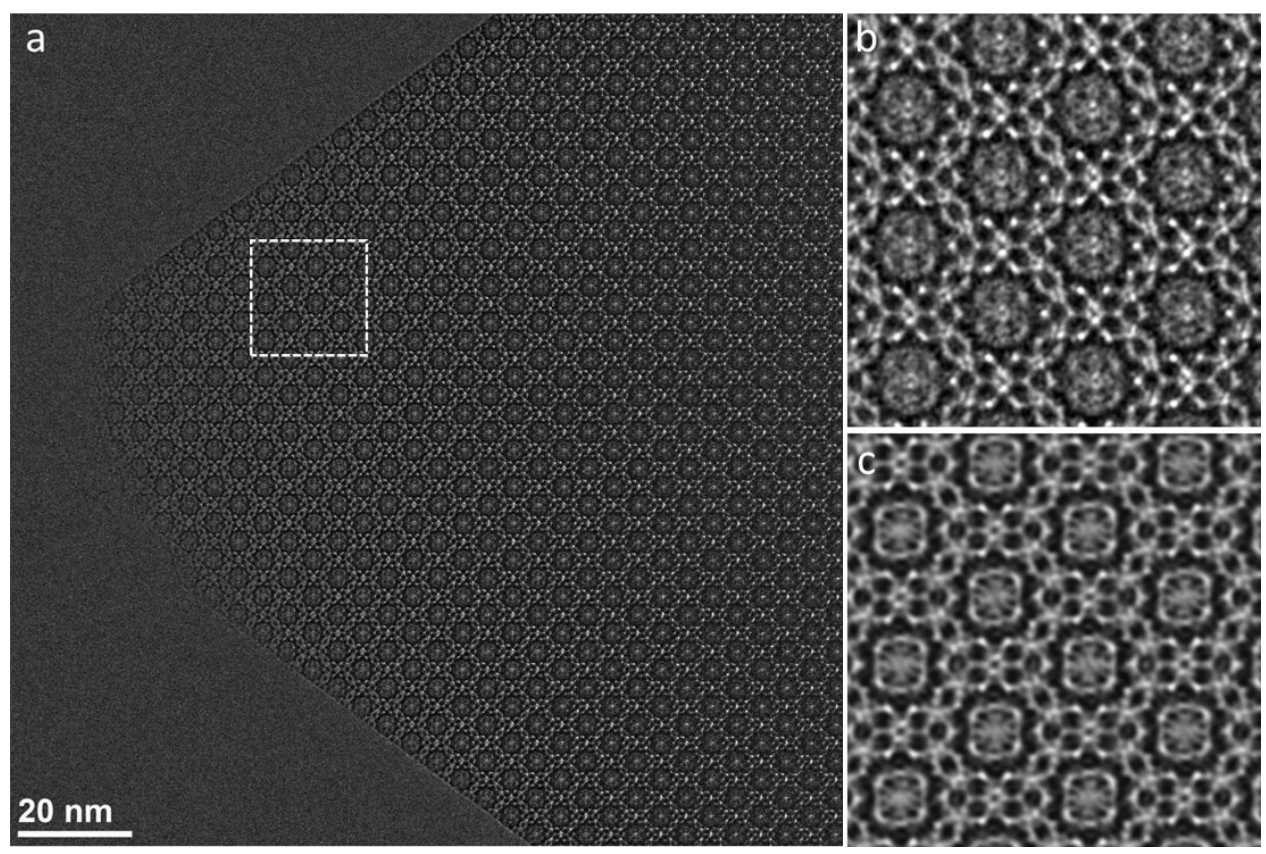

Figure S2. (a) Raw HRTEM image corresponding to Figure 2a in the main text. (b) ABSF-filtered, cropped image from the square area shown in (a). (c) Simulated HRTEM image with QSTEM software (www.qstem.org). Simulation conditions: sample thickness $=62.8 \mathrm{~nm}$, defocus $=-210$ $\mathrm{nm}$, astigmatism $=25 \mathrm{~nm}, 45$ degree, spherical aberration $=0.01 \mathrm{~mm}$. Note that the defocus and astigmatism values are determined using the method described in our previous publication (Science 2018, 359, 675). 


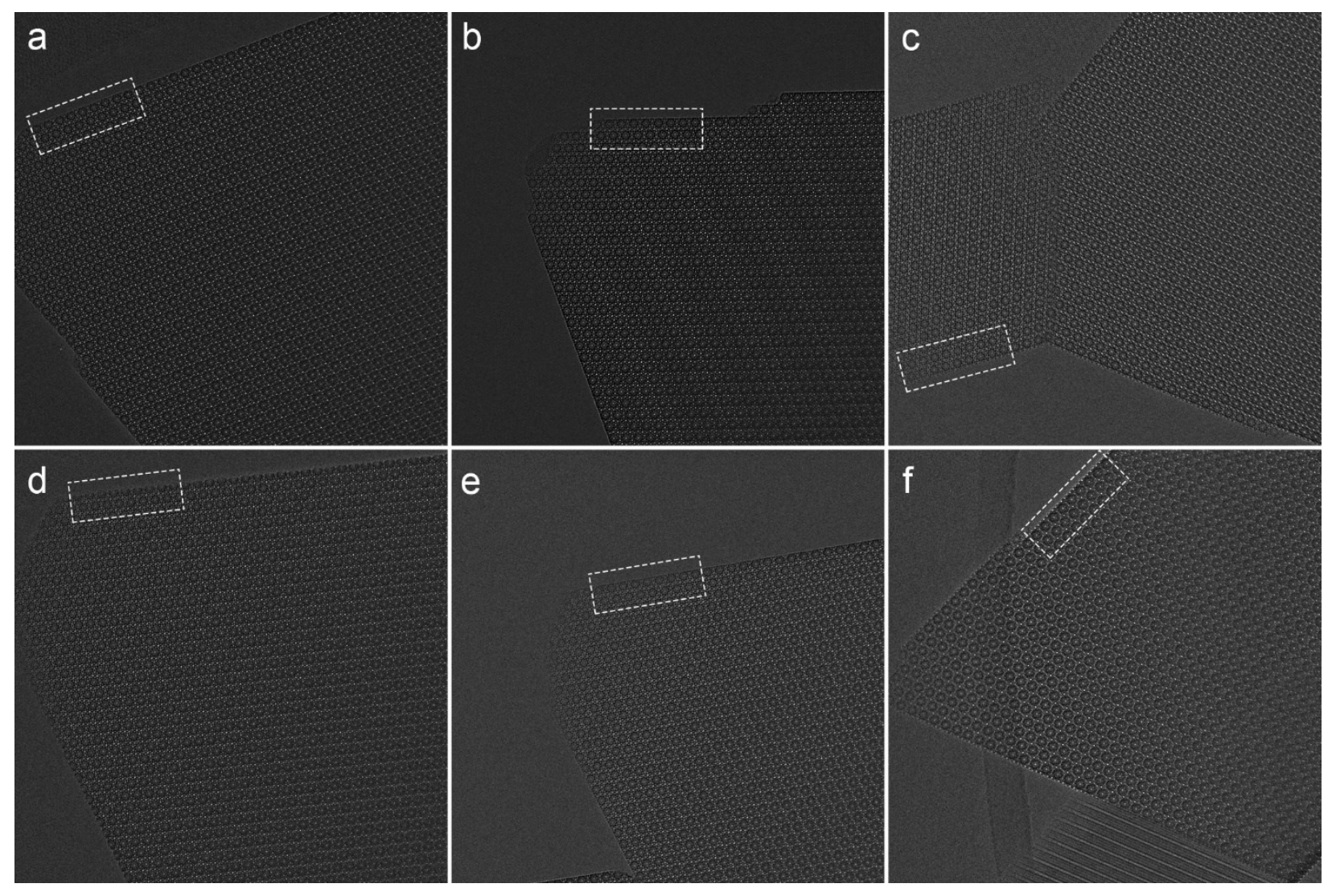

Figure S3. Raw HRTEM images of various MIL-101(Cr) samples. These raw images (a-f) have one-to-one correspondence to the images shown in Figures 3a-3f in the main text that are cropped from the raw images and processed by CTF-correction. The dashed rectangles indicate the selected regions that are presented in Figures 3a-3f. 

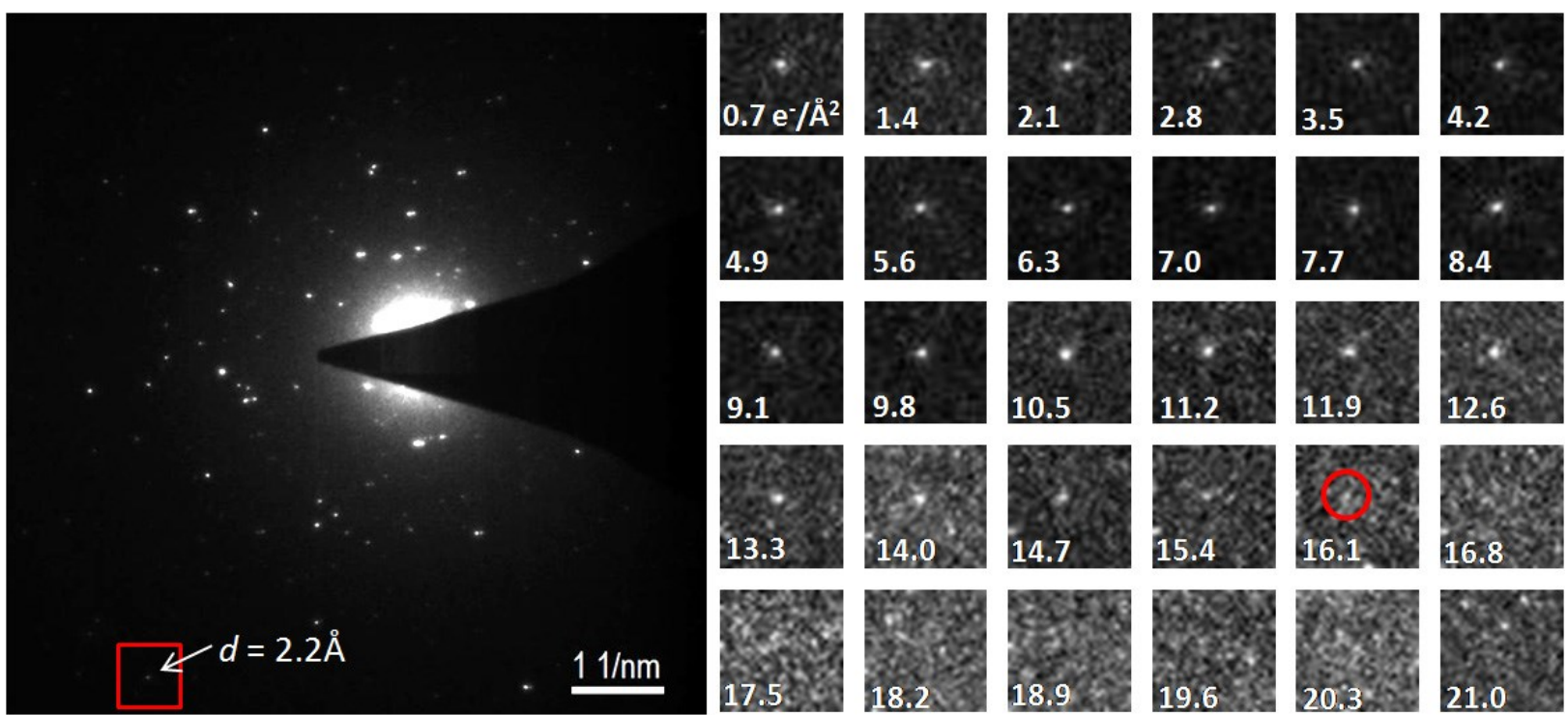

Figure S4. Stability evaluation of MIL-101(Cr) under $300 \mathrm{kV}$ electron beams. Selected area electron diffraction patterns were continuously acquired with an electron dose rate of $0.35 \mathrm{e}^{-} / \AA^{2} / \mathrm{s}$ from a number of MIL-101(Cr) crystals. One of the reflections with a $d$-spacing of $\sim 2.2 \AA$ is marked in the pattern, and highlighted at the right side with the cumulative electron dose labeled. The intensity of marked reflection is disappeared at a cumulative electron dose of $16.1 \mathrm{e}^{-} / \AA^{2}$, which therefore represents a threshold of beam-induced structural damage for this particular MOF. 


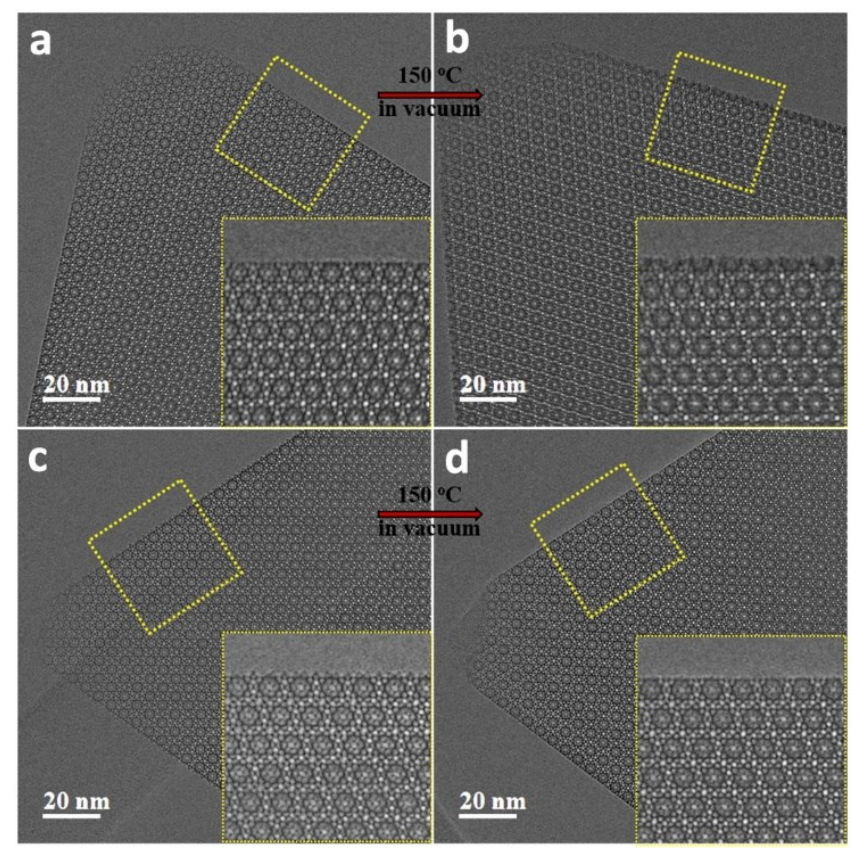

Figure S5. (a-b) HRTEM images of MIL-101-NaF (synthesized with NaF as additive): (a) freshly synthesized and (b) vacuum-heated samples. (c-d) HRTEM images of MIL-101-HCl (synthesized with $\mathrm{HCl}$ as additive): (c) freshly synthesized and (d) vacuum-heated samples. Inset of each panel shows the enlarged image of the highlighted (by dashed square) region that has been rotated to uniform the orientation and processed by CTF-correction. The vacuum heating was conducted at $150{ }^{\circ} \mathrm{C}$ for $24 \mathrm{~h}$. 


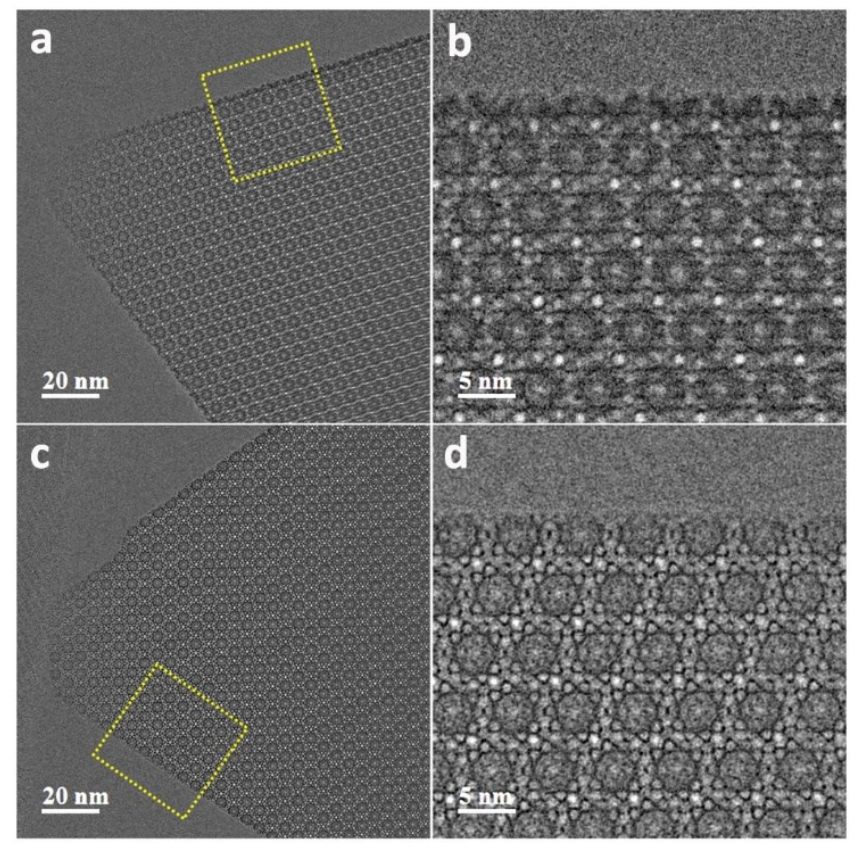

Figure S6. HRTEM images of (a) MIL-101-NA and (c) MIL-101-Ac after a vacuum heating treatment at $200{ }^{\circ} \mathrm{C}$ for $24 \mathrm{~h}$. (b) and (d) show enlarged CTF-corrected images of the regions marked with dashed squares in (a) and (c), respectively. The images indicate that in both cases, vacuum heating results in more open surface cages, in comparison with the "freshly synthesized" samples (see Figure 3 in the main text). 

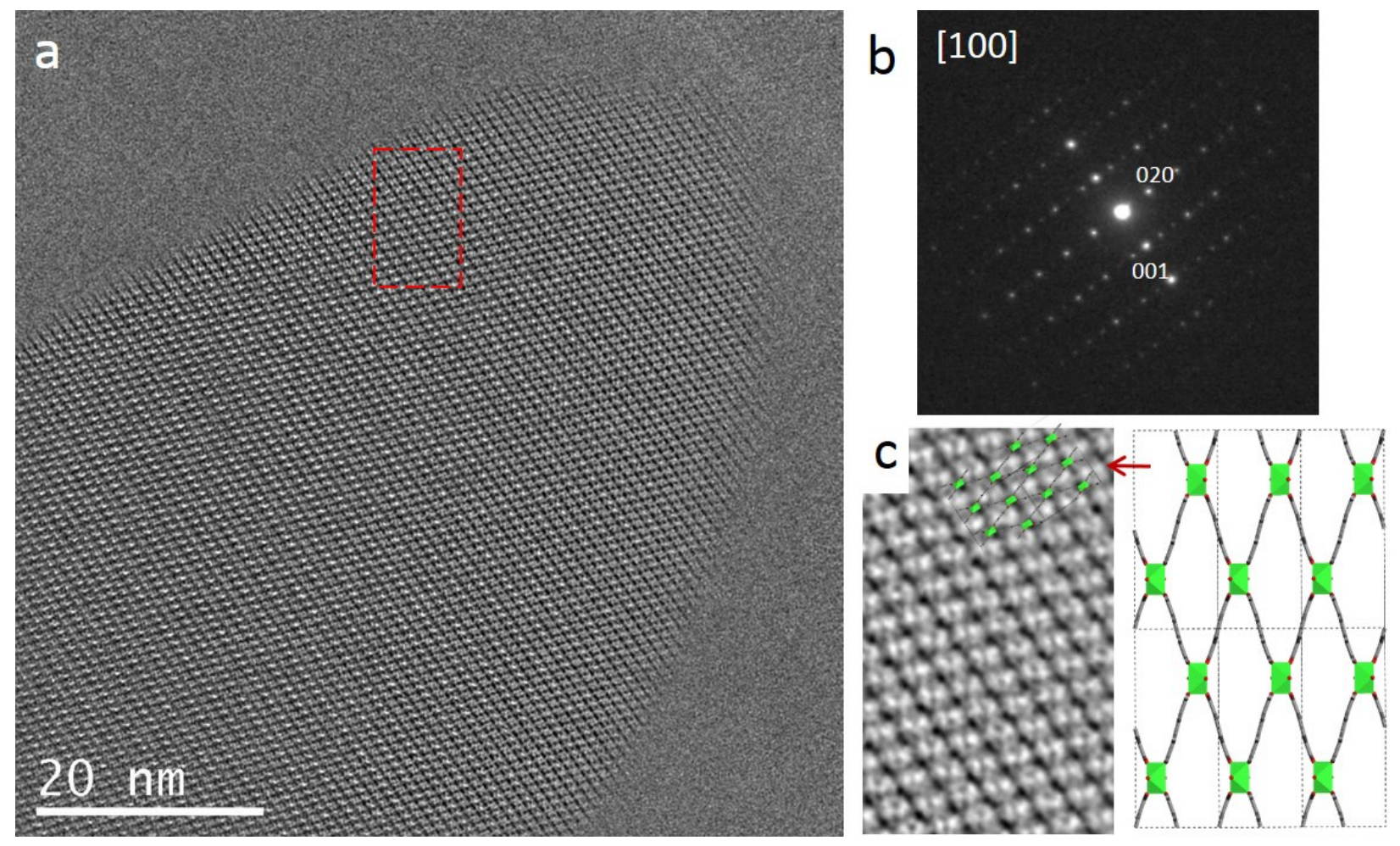

Figure S7. (a) CTF-corrected HRTEM image of a rod-like MIL-53(Cr) crystal present in MIL101-HF as impurity; (b) the corresponding SAED pattern of (a); (c) structural model of MIL-53 overlaid on cropped HRTEM image from the marked area in (a). 

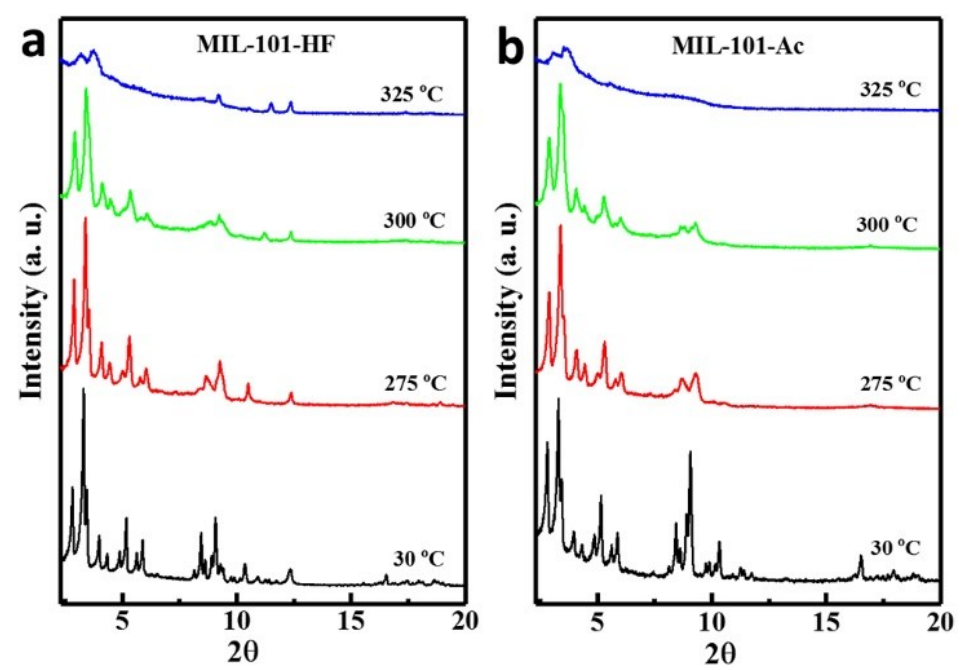

Figure S8. PXRD patterns of (a) MIL-101-HF and (b) MIL-101-Ac heated at different temperatures for $2 \mathrm{~h}$ in $\mathrm{N}_{2}$ atmosphere. 


\section{Computational methods}

\section{Bulk and (111) surface structures of MIL-101(Cr)}

We mainly discuss three MIL-101(Cr) samples, namely MIL-101-HF, MIL-101-NA, and MIL101-Ac; our separate calculation results indicate that MIL-101-NA and MIL-101-Ac have the same framework stoichiometry of $\mathrm{Cr}_{3} \mathrm{O}(\mathrm{BDC})_{3}\left(\mathrm{H}_{2} \mathrm{O}\right)_{2} \mathrm{OH}$, different from that of MIL-101-HF $\left(\mathrm{Cr}_{3} \mathrm{O}(\mathrm{BDC})_{3}\left(\mathrm{H}_{2} \mathrm{O}\right)_{2} \mathrm{~F}\right)$. Therefore, our calculations of surface structures are performed for MIL101-HF and MIL-101-Ac, and we presume the results of MIL-101-Ac apply to MIL-101-NA. The bulk MIL-101(Cr) lattice structures with stoichiometry of $\mathrm{Cr}_{3} \mathrm{O}(\mathrm{BDC})_{3}\left(\mathrm{H}_{2} \mathrm{O}\right)_{2} \mathrm{~F} / \mathrm{OH}$ were constructed with $-\mathrm{F}$ or $-\mathrm{OH}$ randomly attached to one $\mathrm{Cr}$ site of each $\mathrm{Cr}_{3}$ cluster. Due to the large size of the unit cell, we used universal force field (UFF) to describe the bonding interactions among the framework atoms. ${ }^{1}$ We calculated the electrostatic potential within $\mathrm{Cr}_{3} \mathrm{O}(\mathrm{BDC})_{6}\left(\mathrm{H}_{2} \mathrm{O}\right)_{2} \mathrm{~F} / \mathrm{OH}$ clusters which are conventionally considered as SBU of MIL-101. The charge distribution was obtained by fitting the electrostatic potential. Then, we relaxed the unit cells of MIL-101(Cr) (Figure X1) using Forcite within Materials Studio until the change in energy, residue force and displacement of atoms are less than $1 \times 10^{-5} \mathrm{kcal} / \mathrm{mol}, 1 \times 10^{-3}$ $\mathrm{kcal} / \mathrm{mol} / \AA$ and $1 \times 10^{-5} \AA$. The calculated lattice parameters of MIL-101-Ac and MIL-101-HF are 87.89 and $87.91 \AA$, respectively, in reasonable agreement with the experimental value of $\sim 89 \AA$. Different (111) surfaces of MIL-101 were modeled in orthorhombic supercells with vacuum thickness of at least $80 \AA$ (Figures X2 and 3). While constructing the surface models, the surface $\mathrm{Cr}_{3}$ clusters were passivated with protonated BDC to ensure the coordination around $\mathrm{Cr}$ atoms is the same as that in the bulk. The relaxation of surface structures was performed several times to ensure the convergence of the interaction energy within the slab. 
(a)

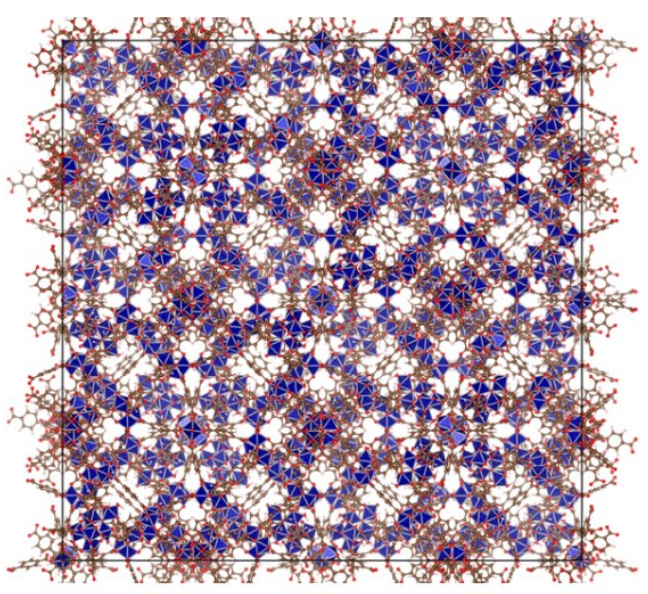

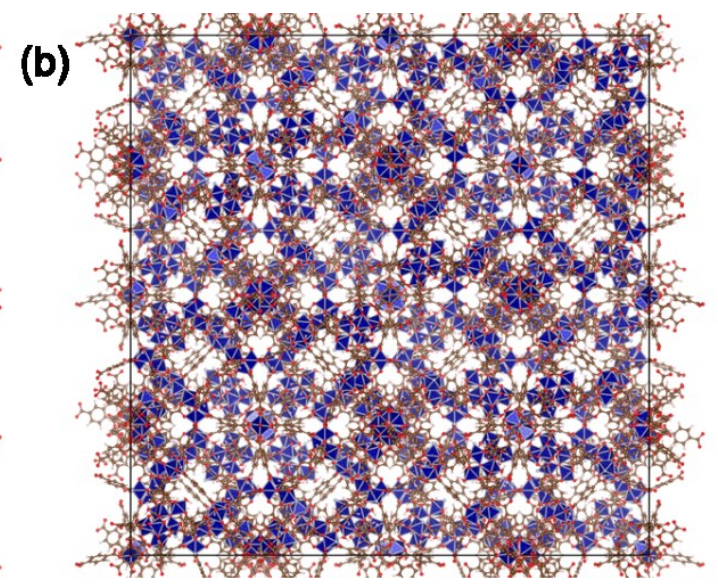

Figure X1. Projected (along the c axis) view of lattice structures of (a) MIL-101-Cr-Ac and (b) MIL-101-Cr-HF. (C: brown; H: pink; O: red; Cr: dark blue; F: light blue.)
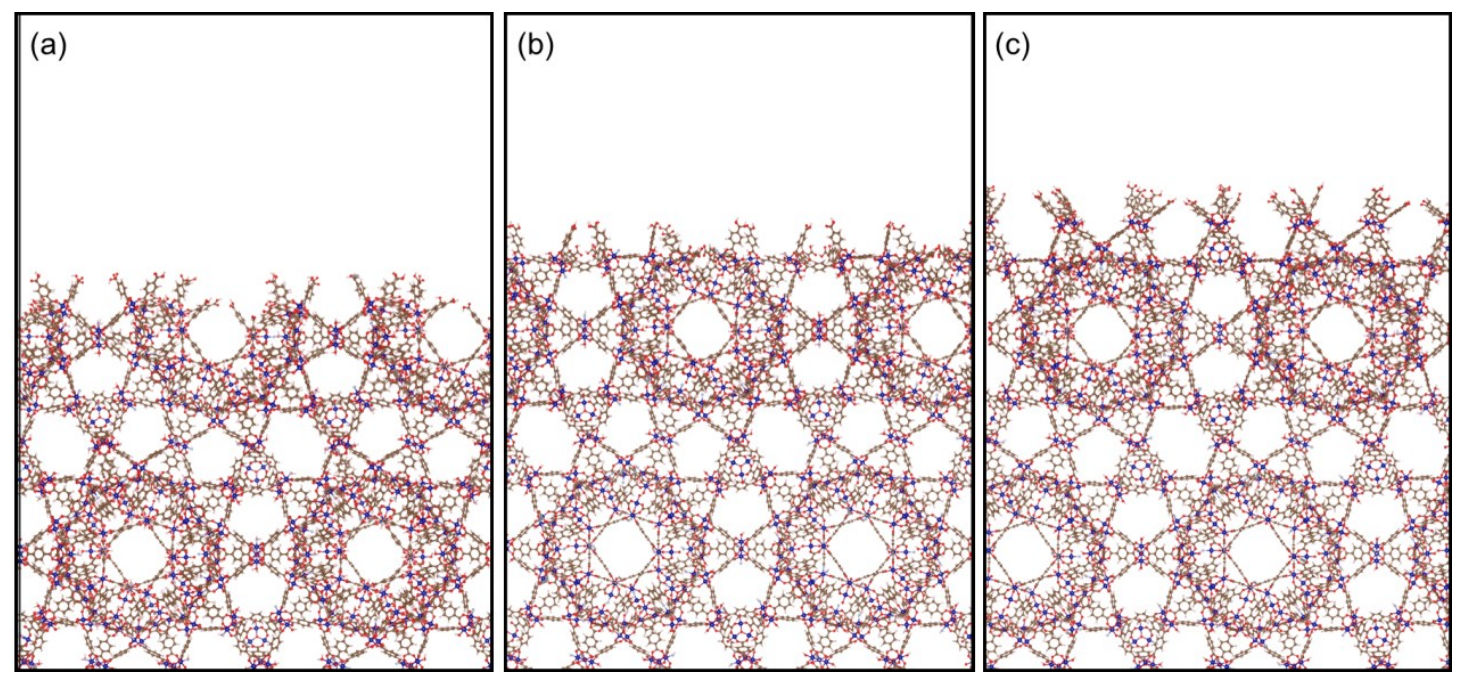

Figure X2. Surface structures of MIL-101-HF with different "completeness" degrees: (a) 60\%, (b) $80 \%$, and (c) 100\% (C: brown; H: pink; O: red; Cr: dark blue; F: light blue). 

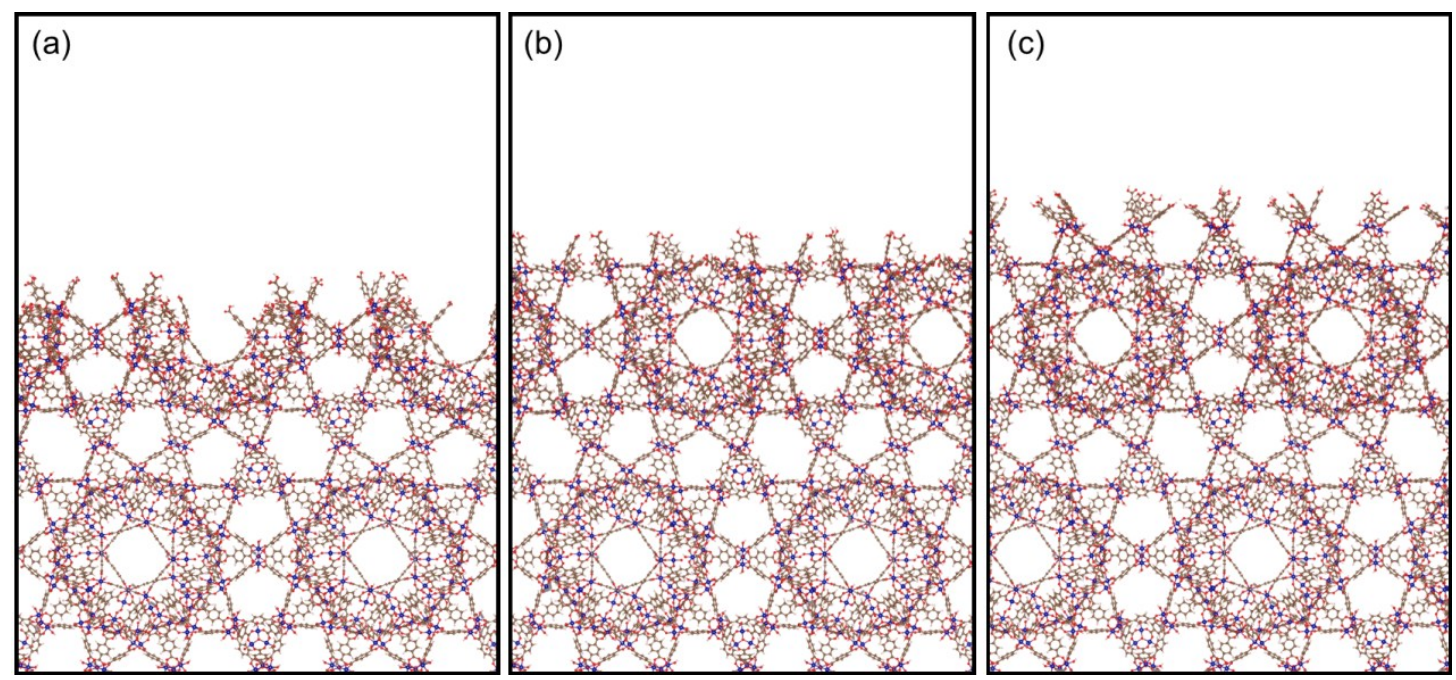

Figure X3. Surface structures of MIL-101-Ac with different "completeness" degrees: (a) 60\%, (b) $80 \%$, and (c) 100\% (C: brown; H: pink; O: red; Cr: dark blue; F: light blue). 


\section{Free energy change for the hydrolysis of $\mathrm{Cr}_{3}$ cluster}

All the first-principles-based calculations were performed using GGA-PBE functional as implemented in $\mathrm{DMol}^{3}{ }^{2-6}$ The electronic states of $\mathrm{C}, \mathrm{O}, \mathrm{F}$ and $\mathrm{H}$, and the valence states of $\mathrm{Cr}$ were treated with DNP basis sets, while the core states of $\mathrm{Cr}$ were handled with DSPP pseudopotentials. ${ }^{7}$ These clusters with stoichiometry of $\mathrm{Cr}_{3} \mathrm{O}(\mathrm{HBDC})_{6}\left(\mathrm{H}_{2} \mathrm{O}\right)_{2} \mathrm{~F} / \mathrm{OH}$ are considered as SBU of MIL-101. ${ }^{8}$ We used one SBU passivated by HBDC to mimic the $\mathrm{Cr}_{3}$ cluster and took the reaction of $\mathrm{H}_{2} \mathrm{O}$ to investigate the impact of $-\mathrm{OH}$ and $-\mathrm{F}$ to the stability of the MIL-101 framework. All the structures were fully relaxed with $\mathrm{DMol}^{3}$ and then subjected to frequency calculations. Considering the complexity of the reactions of the MIL-101 nanostructures that may involve the variation of spin state of the system, we didn't investigate the detailed reaction mechanism. Instead, we passivated the $\mathrm{Cr}(\mathrm{III})$ in octahedral coordination. With this treatment, the reaction of $\mathrm{H}_{2} \mathrm{O}$ with $-\mathrm{BDC}$ or $\mathrm{HBDC}$ is a metathetical reaction with $\mathrm{H}_{2} \mathrm{BDC}$ as the detaching groups. The free energy change ( $\left.\Delta \mathrm{G}_{\text {React }}\right)$ along the proposed reaction paths were calculated with frequency partition functions both reactants and products of the elementary steps.

\section{References}

1. Rappe, A. K.; Casewit, C. J.; Colwell, K. S.; Goddard, W. A.; Skiff, W. M. UFF, A FULL PERIODIC-TABLE FORCE-FIELD FOR MOLECULAR MECHANICS AND MOLECULAR-DYNAMICS SIMULATIONS. $J$. Am. Chem. Soc. 1992, 114 (25), 10024-10035, DOI: 10.1021/ja00051a040.

2. Delley, B. AN ALL-ELECTRON NUMERICAL-METHOD FOR SOLVING THE LOCAL DENSITY FUNCTIONAL FOR POLYATOMIC-MOLECULES. J. Chem. Phys. 1990, 92 (1), 508-517, DOI: 10.1063/1.458452.

3. Delley, B. From molecules to solids with the DMol(3) approach. J. Chem. Phys. 2000, 113 (18), 7756-7764, DOI: $10.1063 / 1.1316015$.

4. Perdew, J. P.; Burke, K.; Ernzerhof, M. Generalized gradient approximation made simple. Phys. Rev. Lett. 1996, 77 (18), 3865-3868, DOI: 10.1103/PhysRevLett.77.3865.

5. Delley, B. Hardness conserving semilocal pseudopotentials. Phys. Rev. B 2002, 66 (15), 155125.

6. Rappoport, D.; Crawford, N. R. M.; Furche, F.; Burke, K. Approximate Density Functionals: Which Should I Choose? In Encyclopedia of Inorganic Chemistry; John Wiley \& Sons, Ltd: 2006; pp 1-14.

7. Inada, Y.; Orita, H. Efficiency of numerical basis sets for predicting the binding energies of hydrogen bonded complexes: Evidence of small basis set superposition error compared to Gaussian basis sets. J. Comput. Chem. 2008, 29 (2), 225-232, DOI: $10.1002 / \mathrm{jcc} .20782$.

8. Cantu, D. C.; McGrail, B. P.; Glezakou, V. A. Formation Mechanism of the Secondary Building Unit in a Chromium Terephthalate Metal-Organic Framework. Chem. Mat. 2014, 26 (22), 6401-6409, DOI: 10.1021/cm5027859. 